\title{
Capital humano y sociedad de control*
}

\author{
Human capital and society of control
}

\section{Capital humano e sociedade de controle}

Fecha de entrega: 2 de noviembre de 2015

Fecha de evaluación: 5 de mayo de 2016

Fecha de aprobación: 2 de junio de 2016

\section{Alejandra Rubio Ríos*}

\section{Resumen}

Partiendo de la noción de "arte de gobierno liberal", que encuentra su configuración en la forma de una Economía Política y su modelo normativo en un gobierno frugal, pretendemos elevar nuestro estudio hacia un análisis del tipo de subjetividad que funciona como correlato de la racionalidad política (neo)liberal: el homo oeconomicus y su actualización en la forma de sujeto “empresario de sí mismo”. Para tal fin nos veremos en la necesidad de señalar la mutación epistemológica planteada por el análisis neoliberal -iniciado por la Escuela de Friburgo y, más específicamente, desarrollado por la Escuela de Chicago en el ámbito norteamericano-, el cual tiende un puente entre el análisis de los procesos económicos y el estudio de la racionalidad interna del comportamiento humano. Asimismo, haremos una incursión en la

* DOI: http://dx.doi.org/10.15332/s0120-8462.2016.0115.04

* Máster en Estudios Avanzados de Filosofía por la Universidad Complutense de Madrid, actualmente cursando el programa oficial de doctorado en Filosofía por la Universidad de Granada, c/lsla Tago Mago, Mazarrón, Murcia, España, 30868. Correo electrónico: alejarub@ucm.es 
teoría del Capital Humano a través de intelectuales como Theodore W. Schultz y Gary S. Becker y sus derroteros actuales.

Lejos de iniciar una relectura en términos puramente académicos, con nuestro análisis -y siguiendo un método praxeológico- pretendemos llamar la atención sobre una de las numerosas vías de investigación que Foucault dejó abiertas. Con esto pretendemos abrir el espacio para un ejercicio crítico que tome por objeto las derivas de esta comprensión de los sujetos en términos de "riqueza" en ámbitos de la vida que no son estrictamente económicos.

Palabras clave: neoliberalismo, homo oeconomicus, sujeto "empresario de sí mismo", capital humano, empresa, inversión.

\section{Abstract}

Bringing into focus the idea of "liberal rationality of government", wich is configurated by a Political Economy form and a frugal government, the aim of this paper is to present our study in the direction of an analysis of the kind of subject wich is working as a correlative part of the (neo)liberal political rationality: the homo oeconomicus and his update in the way of a subject as "expert of himself". This paper critically examines the mutation of the epistemology suggested by the neoliberal analysis originated by the Freiburg School and, specifically developed by the Chicago School. The liberal analysis makes a connection between the analysis of the economics process and the study of the rationality of the human behaviour. Addiotionally, we will do a incursion in the Human Capital Theory following some intelectual figures like Theodore W. Schultz and Gary S. Becker.

It is not our purpose to do a reading in a academic terms; the aim of this paper is to emphasize one of the research line wich was opened by Foucault. Following a practical method, the study has gone some way towards enhancing our understanding of the effects of this type of representation of the subject in terms of source of wealth.

Keywords: Neoliberalism, homo oeconomicus, subject as "entrepreneur of the self", human capital, enterprise, investment. 


\section{Resumo}

Partindo da noção de "arte do governo liberal" que encontra sua configuração na forma de uma Economia Política e seu modelo normativo em um governo frugal, pretendemos levar nosso estudo para uma análise do tipo de subjetividade que funciona como correlato da racionalidade política (neo) liberal: o homo economicus e sua atualização na forma de sujeito "empresário de si mesmo".

Para esse objetivo precisamos assinalar a mutação epistemológica apresentada pela análise neoliberal - iniciada pela Escola de Friburgo e mais especificamente, desenvolvida pela Escola de Chicago no âmbito norte americano-, o qual estabelece uma relação entre a análise dos processos econômicos e o estudo da racionalidade interna do comportamento humano. Também, faremos uma incursão na teoria do Capital Humano a traves de intelectuais como Theodore W. Schultz e Gary S. Becker e suas rotas atuais.

Longe de iniciar uma releitura em termos puramente acadêmicos, com nossa análise - e seguindo um método praxeológico - pretendemos chamar a atenção sobre uma das inúmeras vias de pesquisa que Foucault começou. Com isso pretendemos abrir o espaço para uma atividade crítica que tome por objeto as derivas de esta compreensão dos sujeitos em termos de "riqueza" nos ambitos da vida que não são estritamente econômicos.

Palavras-chave: Neoliberalismo, homo oeconomicus, sujeito "empresário de si mesmo", capital humano, empresa, investimento.

Tomando como eje central los estudios de Michel Foucault sobre la problemática de la biopolítica y el liberalismo, desplegados especialmente en el Curso del Collège de France de 1979 (nacimiento de la biopolítica), aspiramos en este artículo a desarrollar una reflexión en torno a la nueva comprensión de la realidad propia de la racionalidad neoliberal y las mutaciones teóricas que ha conllevado referentes al ámbito laboral. 
Partiendo de la noción de "arte de gobierno liberal", que encuentra su configuración en la forma de una Economía Política y su modelo normativo en un gobierno frugal, pretendemos elevar nuestro estudio hacia un análisis del tipo de subjetividad que funciona como correlato de la racionalidad política (neo)liberal: el homo oeconomicus, y su actualización en la forma de sujeto "empresario de sí mismo". Para tal fin nos veremos en la necesidad de señalar la mutación epistemológica planteada por el análisis neoliberal -iniciado por la Escuela de Friburgo y, más específicamente, desarrollado por la Escuela de Chicago en el ámbito norteamericano-, el cual tiende un puente entre el análisis de los procesos económicos y el estudio de la racionalidad interna del comportamiento humano. Asimismo, haremos una incursión en la teoría del Capital Humano a través de intelectuales como Theodore W. Schultz, Gary S. Becker y sus derroteros actuales. Con esto pretendemos abrir el espacio para un ejercicio crítico que tome por objeto las derivas de esta comprensión de los sujetos en términos de "riqueza" en ámbitos de la vida que no son estrictamente económicos.

\section{Los gobernados: la empresa humana}

Con la problematización de la población como objeto político se traslada el acento que anteriormente recaía en el complejo jurídico-político como instancia de ordenamiento y de control, para situarlo ahora en el ámbito articulado en torno al discurso sobre la riqueza: la Economía como ciencia. Una vez que la Economía se constituye como clave de bóveda del ordenamiento político y social, es cuestión de tiempo -el tiempo ajustado a la lógica y las leyes propias del mercado- que el orden económico y su resorte, el homo oeconomicus, hagan "caducar" al poder soberano (Foucault, 2009, p. 288). La progresiva liberalización del mercado, la defensa de valores liberales tales como la libertad y la dignidad personal del individuo, la desterritorialización de las relaciones económicas y la borradura de las fronteras geográficas componen el suelo de esta neoliberalización de la sociedad.

La sociedad neoliberal no está exenta, sin embargo, del intervencionismo del gobierno. Verlo desde esta óptica supondría caer en el entramado urdido por las relaciones de poder características de la doctrina neoliberal. Hay que destacar y especificar el rostro de este intervencionismo que encuentra su nuevo punto de aplicación en un nuevo territorio definido y conformado a partir del modelo capitalista: la sociedad civil (Foucault, 2009, p. 291). Puesto que el liberalismo clásico y el neoliberalismo forjan su doctrina en la oposición a todo tipo de intervención estatal en lo económico, es 
necesario dar cabida a ciertos mecanismos de control y de regulación que posibiliten y garanticen la consumación de sus fines. El carácter estratégico de este nuevo arte de gobernar queda respaldado por la sutilidad del poder que actúa, no ya sobre los individuos como sujetos de derecho, sino sobre los individuos como sujetos subordinados a sus intereses, o más bien sujetos que se conciben y se constituyen como “empresarios de sí mismos" insertos en la sociedad de empresa (Foucault, 2009, p. 161).

En este trabajo de reflexión nos proponemos exponer algunos de los efectos que comporta el diagrama del poder de la racionalidad neoliberal, tomando como escenario la bisagra que articula la economía y los fenómenos sociales (Foucault, 2009, p. 238). El desplazamiento del intercambio a la competencia como principio del mercado, el abandono del laissez-faire como clave de su funcionamiento, la íntegra superposición de la política gubernamental y los mecanismos de mercado ajustados a la competencia constituyen la materia de una nueva idea: la economía de mercado es un efecto deseable que se ha de conseguir mediante la puesta en marcha de un intervencionismo jurídico del Estado (Foucault, 2009, p. 178). La cuestión estriba, pues, no en el grado de este intervencionismo promulgado, sino en su naturaleza, en su estilo (Foucault, 2009, pp. 130-141).

Tomando como base la caracterización de la sociedad aportada por Hayek (1976) como “orden espontáneo" (p. 36), pretendemos derivar en una reflexión acerca de un nuevo saber (y un nuevo objeto de estudio) que tiene su génesis en la problematización de ciertos dominios a cargo de la economía. La sociedad, dice Hayek, no es ni puede ser un sistema racionalmente organizado. La sociedad, como proceso dinámico en constante evolución cuyo motor es la innovación y la generación continua de información, resiste a todo intento de organización voluntarista (Huerta, 1997, p. 135) ${ }^{1}$. Los grandes sistemas que antaño organizaran las relaciones entre los integrantes de una comunidad con arreglo a categorías metafísicas se han evaporado. Derribados esos pilares divinos, de lo que se trata ahora es de construir un nuevo sistema-que

1 El "error intelectual" que los pensadores neoliberales denuncian en la pretensión de elaborar una ingeniería social, entronca con la incompatibilidad esencial entre "la multiplicidad no totalizable, característica de los sujetos de interés" y "la unidad totalizadora del soberano jurídico" Foucault, M. (2009, p. 281), NBP, Buenos Aires: FCE. Hemos incidido en el término "proceso" en tanto que el estilo neoliberal de gobierno se caracteriza como un gobierno de procesos; Vázquez, Francisco, "empresarios de nosotros mismos". Biopolítica, mercado y soberanía en la gubernamentalidad neoliberal. (2005, p. 84). En Ugarte, J. (Ed.). La administración de la vida. Estudios biopolíticos. Barcelona: Anthropos. 
es también moral- no ya adscrito a una supuesta sabiduría divina, sino a un saber que es técnico y pragmático.

Es aquí donde, a propósito de la borradura de los límites entre política y economía, esta va a definirse como una ciencia del comportamiento humano. Un saber específico que toma como objeto de estudio un dominio muy particular: un saber de lo humano cifrado en el esquema de principios-fines y codificado por una racionalidad que evalúa los medios (Mises, 199, p. 201). La razón neoliberal desborda el ámbito de una mera evaluación de los medios. En efecto, esta evaluación requiere la interferencia de ciertos valores correspondientes a un determinado proyecto de sociedad. La consumación de la utopía liberal necesita de una política activa e interventora que oriente, conduzca y conforme la actividad de los hombres (Harvey, 2011, pp. 65-66).

En estrecha conexión con la emergencia de este saber se configura con el neoliberalismo una relación renovada entre el poder y el saber de los expertos. Puede decirse que el lazo poder-saber-verdad se hilvana con el propósito de, en la reconceptualización del sujeto gobernable, asegurar la eficacia de un poder que se aplica sobre una realidad heterogénea. En otras palabras, se trata de un instrumento de la racionalidad neoliberal que busca gestionar los riesgos y las libertades que comporta la sociedad contemporánea. A partir de la observación del funcionamiento del mecanismo del engranaje neoliberal podemos deducir en qué consiste la sociedad de control.

El proceso de desestatalización de la sociedad se desarrolla conjuntamente con un proceso de extensión y difusión de ciertos saberes que, en una forma institucional o informal, van a fijar sobre los cuerpos la condición dócil, flexible, que requiere el sistema para funcionar. En el afán del neoliberalismo por presentarse como la única alternativa, estos saberes van a definirse como grey sciences (Rose, 1996, p. 56). Estos saberes articulados por el modelo del cálculo económico pretenden instalar sus categorías propias en la base de la comprensión del comportamiento humano de unos sujetos intensamente activos. Los "expertos de la subjetividad" logran, con el uso de ciertos mecanismos, la extensión de las metas políticas, sociales y económicas hacia el dominio personal de los compromisos, preferencias y decisiones del individuo (Rose, 1996, p. 58). La base de una sociedad sana queda asegurada con la cultura de autoprovisión y la autoresponsabilidad en el seno de un "orden espontáneo"2.

2 "El individuo y su sentido de la responsabilidad constituyen el resorte maestro secreto de la sociedad", Röpke, Wilhem, Estado benefactor e inflación crónica [trad. al español]. A Humane Economy: The 


\section{Sociedad de empresa y ethos empresarial: el sujeto "empresario de sí mismo"}

El "gobierno a distancia” constituye el rasgo de identidad en el diagrama del poder contemporáneo en la sociedad de los competentes (Rose, 1996, p. 43). Es la "disciplina impersonal del mercado" la que conforma este tipo de sociedad donde los sujetos son $\mathrm{y}$ desean ser intensamente productivos en el juego del mercado:

La disciplina impersonal del mercado a menudo se basta a sí misma para asegurar un grado suficiente de conformidad incluso con los decretos más ásperos y letales del mismo mercado. Solo cuando los afectados se organizan para rechazar las exigencias de reducir su consumo y aceptar las consecuencias [...] se hace necesario el suplemento de la fuerza (Montag, 2006, p. 28).

La razón de gobierno neoliberal va a intentar, pues, que no se alcance ese punto limítrofe entre el sujeto gobernable-dócil-satisfecho y el sujeto rebelde. Se ha de implantar en los individuos el deseo de formar y tomar parte en la economía de mercado, sin importar los efectos que esta disposición de la sociedad opere sobre los individuos. En otras palabras, "los cambios a los que la gente debe someterse son parte del costo del progreso [...]" (Hayek, 1981, p. 25). Este sometimiento de los individuos va a definirse por una dulce envoltura que promete a todos y cada uno la posibilidad de autorrealizarse en el interior de un proyecto de vida que traerá consigo la mejora de la situación personal en la sociedad. La racionalidad del sujeto así descrito estriba en la orientación de las decisiones en la dirección que en cada momento resulte adecuada para prosperar de un modo indefinido; esta condición ha sido planteada por los pensadores neoliberales como una tendencia innata que ningún tipo de censura moralista puede acallar (Mises, 1995, pp. 190-191).

En esta senda, el análisis económico del comportamiento humano toma como presupuesto esta modalidad de sujeto: el homo oeconomicus. El sujeto así definido se caracteriza por una conducta finalista que se le supone connatural. Sus acciones en el marco de la economía del mercado se ven reducidas a la posibilidad de elección

social framework of the free market. Chicago: Henry Regnery Company, A Gateway Edition, 1971, pp. 210-211 y p. 222. Asimismo Hayek, Friedrich, A., Los Fundamentos Éticos de una Sociedad Libre, Ciclo de conferencias sobre Fundamentos de un Sistema Social Libre, Santiago de Chile, Centro de Estudios Públicos, 1981, p. 135. 
estratégica de medios, vías e instrumentos, siendo la condición inicial la escasez de medios y la presencia de fines competitivos (Foucault, 2009, p. 265) 3 . No corresponde al saber económico una condición estable por cuanto su objeto de estudio revela una condición dinámica. En efecto, el comportamiento humano y las relaciones que emergen e implosionan en el seno de la sociedad poseen una materia en continua transformación, y de lo que se trata con el análisis económico es de conocer el cómo y los efectos de esta red de interrelaciones humanas. En este sentido, la economía como ciencia desborda su dominio tradicional, y lo que la distingue del resto de ciencias es la forma de analizar las actividades humanas ${ }^{4}$.

Puesto que la intervención gubernamental neoliberal se aplica sobre el marco, este saber económico del comportamiento humano va a fijar su atención en aquellos hechos que representen una suerte de respuesta estable por parte de los sujetos ante las diversas conjugaciones posibles de las variables del medio con la finalidad de conocerlas y, además, de implementar otras variables de comportamiento a partir de ciertas técnicas. El homo oeconomicus, sujeto "eminentemente gobernable", asume el papel que le corresponde en el escenario económico ${ }^{5}$.

Hay que poner de relieve lo siguiente: el tránsito del liberalismo clásico al neoliberalismo implicó una mutación sustancial en la concepción del homo oeconomicus que no debe pasar desapercibida. En la sociedad hecha a imagen y semejanza de la economía de mercado de competencia, el sujeto que habita en ella no puede seguir siendo el hombre del intercambio equitativo; por el contrario, y en respuesta a las exigencias de esta nueva realidad, se trata del sujeto económico productor y

3 Foucault localiza la aparición de este homo oeconomicus como correlato del arte de gobernar liberal a partir del problema que supone la incompatibilidad entre el sujeto de derecho y el sujeto económico, $p$. 290, cfr. Matsusaka, John G., "The economic approach to democracy", en Mariano Tommasi, Kathryn lerulli (Eds.). The new economics of human behaviour, Cambridge, Cambridge University Press, 1995, p. 141.

4 "Mi labor investigadora utiliza el enfoque económico para analizar cuestiones sociales no consideradas normalmente por los economistas". Febrero, R., y Schwartz, P. (Eds.). La visión personal de Becker. El discurso del Nobel: El modo económico de analizar el comportamiento, La esencia de Becker. Barcelona: Ariel, 2001, p. 605.

5 "[...] La economía así entendida podrá definirse entonces como la ciencia de la sistematicidad de las respuestas a las variables del medio", Foucault, M., NBP, op. cit., pp. 266-267, cfr. Rose, N., Governing "advanced" liberal democracies", en Barry; Osborne: Rose, Foucautl and political reason. Liberalism, neoliberalism and rationalities of government: London: UCL Press, 1996, p. 46, sobre la asunción voluntaria de las obligaciones y las libertades individuales. 
empresario $^{6}$ (Foucault, 2009, p. 158). Observamos los efectos de este tránsito en los estudios de Gary Becker cuando integra en su enfoque la teoría de la producción y de la inversión a la teoría de consumidor. La reformulación y revalorización de la noción de "inversión" constituye un hito en el desarrollo de las teorías económicas contemporáneas; comprender la figura del consumidor en relación directa con la del productor y el inversor es lo que permite sostener que todo agente social implicado en el juego económico es productor de bienes básicos e inversor en estos. En otros términos, es el hombre cuya esencia reside en la función empresarial. Por lo tanto, frente a la sociedad jurídica, la sociedad de empresa y, frente al sujeto económico del intercambio, el sujeto productor y empresario.

Lo que ha acontecido en las últimas tres décadas refiere a un proceso de generalización de la forma empresa, de tal modo que desde la corporación más compleja hasta la unidad más pequeña que forme parte de la sociedad va a disponer de un mismo corpus de oportunidades y obligaciones. Aquí la doctrina neoliberal ha visto incrementada la eficacia de su proyecto: con la homogeneización de las heterogeneidades naturales a la sociedad y la imbricación de las prácticas de poder y las tecnologías del yo se origina, al mismo tiempo, una cultura y un ethos empresarial. La ética empresarial actúa como una tecnología más en el engranaje neoliberal, determinando la sustancia ética del individuo al mismo tiempo que un modo de sujeción. Una vez sembrados ciertos valores e instalados ciertos hábitos, el Estado ve reducida su tarea de vigilancia y control extremos que era característica (y necesaria) en los albores de la producción capitalista. Al mismo tiempo, la transmutación del homo oeconomicus en sujeto empresario y productor -que es presentada como un desplazamiento natural- responde en realidad a unas acciones gubernamentales que, en la programación del entorno, hace lo mismo con el individuo. Queremos detenernos en esta idea con el objetivo de esclarecer lo que se está poniendo en juego con la generalización de la forma empresa y la integración de los individuos en el mercado con base en su función empresarial.

Resulta interesante la observación de los economistas neoliberales según la cual la función empresarial coincide con la acción humana misma. Es decir, somos empresarios en todas y cada una de las actividades que realizamos racionalmente

6 Sobre esta distinción en el contexto de las aportaciones correspondientes al paradigma austríaco y al paradigma neoclásico v. Huerta, J. La Escuela Austríaca: mercado y creatividad empresarial. Madrid, Instituto de Análisis Industrial y Financiero, UCM, 1997, pp. 19-20. 
aprovechando las oportunidades que se nos plantean en nuestro entorno (Huerta, 1997, p. 33). Este resultado no puede sorprender cuando hablamos de la gubernamentalidad liberal. El individuo pasa a ser el responsable de sus decisiones y de la situación personal que deriven de estas. En otras palabras, los principios básicos que sustentan y posibilitan esta nueva ética empresarial son los de autogestión (de la sociedad) y autorresponsabilidad (del sujeto económico) (Vázquez, 2005, p. 83). Con este giro el ideal neoliberal adquiere una mayor sofisticación: el Estado se ve liberado de la obligación de procurar los medios para la subsistencia y el desarrollo de los individuos, ahora autodisciplinados:

El Estado Benefactor ha perdido su razón de ser. El progreso se ha de medir ahora por el grado en que se puede pretender que las grandes masas del pueblo se mantengan con sus propios recursos y bajo su propia responsabilidad, mediante el ahorro y el seguro.

Nuestra regla y norma y nuestro ideal aceptado con alegría deben ser la previsión mediante el esfuerzo y la responsabilidad individuales, complementados con la ayuda mutua. Es el ideal de la "casa en orden” (Röpke, 1971, pp. 202-223).

Según la caracterización de Röpke, es la capacidad de autoprovisión lo que funciona como criterio de racionalidad del sujeto. Esto le lleva a declarar, no exento de osadía, que tal condición racional es la cifra de la autonomía y del grado de madurez del hombre y de una sociedad entera. El progreso pasa, pues, por la eliminación de la "muleta" de la tutela estatal".

El carácter crítico que define a la doctrina liberal respecto al Estado y a todas las variantes de intervencionismo se aplica también en el plano individual sobre los sujetos que integran la sociedad de empresa. Si la economía ha sido definida abiertamente como "una ciencia lateral con respecto al arte de gobernar" (Foucault, 2009, p. 285), ahora se presenta en lo tocante a los individuos mostrando esta misma faceta. La competencia, comprendida como un eidos, ocupa el lugar de un ideal normativo conforme al cual deben actuar y comportarse todos los agentes de la sociedad (Foucault, 2009, p.

7 "[...] El consumidor tiene que haberse procurado previamente, por una u otra via, el dinero que luego destina al consumo y la inmensa mayoría de los consumidores se halla precisamente integrada por los propios trabajadores [...]". Mises, L. von. Sobre Liberalismo y Capitalismo. Madrid: Unión Editorial, 1995, p. 286. 
132). Así, frente al riesgo constante de quedar fuera del selecto club de empresarios, el sujeto va a pasar a ser asediado por ciertos valores y ciertas conductas que ha de implementar en su vida cotidiana:

Es preciso que la vida del individuo se inscriba no como vida individual dentro de un marco de gran empresa que sería la compañía o, en última instancia, el Estado, sino [que] pueda inscribirse en el marco de una multiplicidad de empresas diversas encajadas unas en otras y entrelazadas [...] es menester que la vida del individuo [...] haga de él algo así como una suerte de empresa permanente y múltiple (Foucault, 2009, p. 239)

Foucault tematiza la idea de una reconstrucción de la noción de empresa alrededor de los denominados "valores calientes", siendo el objetivo de esta transfiguración la consolidación de la empresa como el lugar en el que el sujeto interactúa con otros sujetos, y no ya como mero lugar de trabajo (Foucault, 2009, p. 240). Esos ciertos valores y conductas que contribuyen en la producción del sujeto-empresa son implementados a través de un nuevo haz de tecnologías y estilos de gestión propios de la gubernamentalidad liberal. La política laboral y el sistema educativo sirven como ejemplos (Foucault, 2009, p. 91). En el ámbito laboral el sujeto va a encontrar una multitud de empresas que guardan el aspecto de una suerte de "microsociedades", con los elementos propios de una sociedad ${ }^{8}$, logrando eliminar la atmósfera de alienación que tradicionalmente envuelve al lugar de trabajo (Foucault, 2009, p. 240).

Hasta aquí hemos tratado de ilustrar los efectos de la implantación de una economía del mercado. Hemos de completar el planteamiento de la cuestión de la generalización de la forma empresa y la renovada comprensión del individuo como empresario y productor, con un último apunte. La concepción del sujeto económico como empresario en el contexto de una economía de mercado cifrada en la competencia implica, necesariamente, comprender a este como inversor. En otras palabras: como "empresario de sí mismo". Este punto crítico constituye el reverso de la garantía de las libertades que el neoliberalismo presenta como encabezado de su programa. Foucault, en un

8 "[...] Las organizaciones desarrollarán a nivel global sus propios componentes culturales que serán compartidos por todos sus empleados [...]", mediante la creación de "una identidad propia [de los empleados] y un sentimiento de pertenencia [...]", Pascual J. L. Gestión del capital humano en las organizaciones: entre nichos y bichos, ¿valor estructural o valor emocional? Tarragona: Altaria, 2013, pp. 60-61 [ligeramente modificado]. 
intento por hacer visible la letra pequeña que suele pasar desapercibida en la lectura del contrato, expone que esas libertades prometidas van a encontrar su contrapeso en las formas del control, la coacción y la coerción ${ }^{9}$. En definitiva, el gobierno liberal avanzado es un "gobierno por subjetivación” (Vázquez, 2005, p. 94).

El mayor logro del neoliberalismo no reside, pues, en las transformaciones del suelo económico efectuadas, sino en la reconfiguración de la autocomprensión y la autoproducción del individuo así presentado. Estas mutaciones han tenido un calado profundo en la política laboral, en la organización del trabajo y, lo que resulta de mayor interés en el contexto de nuestro estudio, en la propia naturaleza del trabajo.

\section{La teoría del Capital Humano. La Escuela de Friburgo y la Escuela de Chicago}

La emergencia del sujeto económico como "empresario de sí mismo" es coetánea a las novedades que por entonces se planteaban en el plano teórico de los estudios sobre el trabajo. Prueba de ello fue el avance de la economía como ciencia del comportamiento humano. Tomando como axioma la posible mercantilización de todo, los economistas de la época tomaron como foco de sus análisis un recurso que había sido minusvalorado hasta el momento: el capital humano como la mayor fuente de riqueza, y no ya el capital físico. El supuesto subyacente a esta consideración determina que la población es un recurso escaso, cuyo valor viene determinado por su calidad (y no por su cantidad $)^{10}$.

El creciente interés por el comportamiento humano en el mundo laboral se debe a los efectos directos que provocan las acciones de los individuos en el mercado. Por este motivo lo que va a caracterizar a este nuevo capital humano en un mercado cuyo motor es la competencia es la idoneidad. El origen de esta idea puede ser rastreado en el pensamiento económico tayloriano, en el cual se reconoce ya que la productividad del trabajo es un factor más en el desarrollo económico -aunque el desarrollo estuviese

9 "Libertad económica, liberalismo en el sentido que acabo de decir y técnicas disciplinarias: también aqui las dos cosas están perfectamente ligadas", Foucault, Michel, NBP, op. cit., p. 75.

10 La población constituye la nueva "riqueza de las naciones", y por ello será preciso invertir en ella para que vea incrementada su calidad. Como toda inversión, también esta será planteada en términos de oferta-demanda, con el fin de que las ganancias resultantes excedan al coste de adquisición, Schultz T. W. Invirtiendo en la gente: la cualificación personal como motor económico. Barcelona, Ariel, 1985, p. 21. 
cifrado por entonces en la acumulación de capital (Coriat, 2001, pp. 33-34)_- ${ }^{11}$. La teoría neoclásica instala definitivamente la idea de que las variaciones en los resultados de la producción guardan una relación directa con las diversas alternativas en el modo de uso de la mano de obra. El nivel de productividad depende, pues, del modo en el que sean conjugados el capital físico y el capital humano.

El escepticismo generalizado en torno a la consideración de este nuevo capital intangible tenía claros motivos. La teoría económica se encontró con numerosas opacidades y elementos difícilmente integrables al cálculo económico. La manipulación de información y conocimientos pertenecientes a las ciencias humanas inyectaba un componente inestable en la investigación que impedía seguir elaborando los análisis y cálculos como se había hecho hasta el momento. Sin embargo, este factor inestable que atañe al carácter cualitativo de los objetos, dirán los pioneros de la teoría del capital humano, se revela como el más valioso para una economía del mercado de la competencia en el cual actúan e interactúan agentes altamente activos. Los economistas neoliberales dieron cada vez un mayor peso en sus investigaciones a estos niveles microeconómicos y al carácter pragmático del entorno empresarial. La disposición de la sociedad como conjunto de microempresas que intervienen activamente en el juego económico y que actúan como motor de la innovación y de desarrollo, constituye uno de los factores que condiciona el interés desmesurado por el saber sobre los sujetos y sus conductas. La cuestión central pasaba por establecer cómo era posible determinar la rentabilidad de la inversión en recursos humanos. El interés que inspiró los estudios Gary Becker y Theodore Schultz fue rellenar esa "página en blanco" en la que los economistas no habían escrito nada (Foucault, 2009, p. 220) ) $^{12}$.

Como punto de partida de la introducción del trabajo en el análisis económico, los economistas plantearon la pregunta por el trabajo. Hasta el momento, "las teorías del comportamiento de la empresa [...] habían ignorado invariablemente el efecto

11 El proceso de aceptación del valor capital humano en los niveles de productividad fue lento y no estuvo exento de un escepticismo generalizado. Robert Owen, un industrial británico, estableció las bases de lo que más tarde constituiria la filosofía humanística de la empresa, asumiendo la posibilidad de una mayor ganancia a partir de la inversión en recursos humanos que en bienes de capital (físico), v. Morales-Arce, R. En torno al capital humano: aspectos básicos en su consideración empresarial. Madrid, Centro de Estudios Ramón Areces, 2005, p. 35.

12 Sobre la sobrevaloración del capital físico y el ascenso de la importancia del capital humano: "el futuro de la humanidad no está ordenado de antemano por el espacio, la energía y la tierra cultivable. Estará determinado por la evolución inteligente de la humanidad". Schultz, T. W. Invirtiendo en la gente. Barcelona, Ariel S. A., 1985, p. 15. 
del propio proceso productivo sobre la productividad del trabajador" (Becker, 1983, p. $28)^{13}$. El trabajo, dicen, no puede seguir siendo comprendido a partir de las categorías clásicas que lo definen a partir de la noción de proceso. El trabajo ha de ser concebido ahora como una actividad, en la que el sujeto económico integrado en el mercado (como empresario y empresario de sí mismo) realiza de un modo racional-estratégico (Foucault, 2009, p. 225). Con este cambio en la perspectiva del análisis, estos estudiosos pretenden extraer la esencia del trabajo a partir de la vivencia de aquel que trabaja. La vivencia del trabajador nos dice que el motivo que le impulsa a trabajar es el de generar un flujo de ingresos (no ya un salario) que le permita seguir completando el proceso de autodesarrollo. Con estos desplazamientos cambia por entero la comprensión (y la autocomprensión) del sujeto que trabaja: este, como empresario de sí mismo, no vende su trabajo; oferta un plexo de rasgos que corresponden a la idea normativa que se implanta -y que es condición de posibilidad al mismo tiempo- con el proceso de neoliberalización de la sociedad.

Afirmar que el sujeto empresario se oferta a sí mismo en el mercado laboral saca a relucir, por fin, el rasgo de descualificación que paradójicamente le caracteriza. Bajo el imperativo de la productividad, la eficacia y la rentabilidad, el capital que comporta el sujeto se comprende en el marco de la teoría de capital humano bajo la misma luz en que lo fuera el capital físico. Huelga decir que el valor de este capital humano, representado en la economía de mercado por su intangibilidad, va a ser considerado en su aspecto cualitativo. Diluyendo las reticencias de aquellos que muestran reticencias ante el capital humano, finalmente se hizo posible cuantificar su valor económico a partir de un criterio central: la idoneidad (Foucault, 2009, p. 226). A este respecto, Schultz advierte del error generalizado por el que se ha mantenido el supuesto de la homogeneidad del capital. Cada capital tiene sus especificidades, y la posibilidad de apreciar el modo en que el capital humano contribuye en la productividad solo se puede plantear una vez reconocida la lógica y el ritmo de las inversiones de este tipo (Schultz, 1985, pp. 20-21). "Ser idóneo", reflejar esta idoneidad en el mercado, solo puede significar lo siguiente: el sujeto ha de resultar "atractivo" ante el ojo avizor de los empleadores dispersos en la sociedad de empresa ${ }^{14}$.

13 Ligeramente modificado. Cursiva propia.

14 Esta cuestión entronca directamente con el estudio llevado a cabo por Becker en torno a la formación general y la formación especifica. La tasa de rotación depende exclusivamente del interés que la empresa vierte en sus empleados; asi, un trabajador que posea una formación específica tendrá un mayor valor que uno que tenga una formación general. Becker, G. S. El capital humano, op. cit., p. 44-45. "Una empresa estaría dispuesta a pagar por la inversión en capital humano que realizan sus 
Como correlato de la idoneidad existe otro valor de igual peso: la empleabilidad. Lo más importante queda así registrado en el rasgo formal de estos valores y en la flexibilidad absoluta de la materia que pretenden informar. La empleabilidad del sujeto constituye un fiel reflejo del nivel de probabilidad de aceptación y contratación para desarrollar determinadas actividades laborales ${ }^{15}$. Hay que hacer notar que estos valores solo adquieren su sentido pleno sobre la base de una radicalización de la competencia. La empleabilidad refuerza el imperativo de la autorrealización y de la idoneidad, y esto es así en la medida en que, abolido el derecho mínimo a la existencia el sujeto se ve empujado a una renovación incesante de sus aptitudes. Así pues, el sujeto ha de vivir "ampliando frecuentemente sus originarias habilidades o incluso adquiriendo nuevos conocimientos. [...] pues así lo exige la realidad diaria” (Mises, 1995, p. 92).

Foucault incide sobre un aspecto que es relevante en su análisis genealógico del sujeto moderno. Una vez más, la extensión de la economía fuera de sus dominios trae consigo unos efectos que sobrepasan lo económico. En efecto, con la finalidad de adquirir un reflejo atractivo en el mercado laboral, el sujeto se ve empujado a incrementar sus niveles de idoneidad. El rasgo diferencial del capital humano estriba en que, a diferencia de un bien físico que puede poseerse, perderse y recuperarse, este se encuentra integrado en el sujeto mismo y es inalienable (Becker, 1983, p. 114). Del mismo modo en que se invierte en una infraestructura para mejorarla, el sujeto empresario ingresa en una dinámica que le insta a hacer ciertas inversiones en sí mismo, valorando el costo y el rendimiento de sus decisiones ${ }^{16}$. El incremento del propio capital humano (factores físicos y psicológicos) dependerá, pues, de las decisiones autónomas y personales que cada sujeto, como agente económico y productor, tome en relación con las posibles inversiones en sí mismo que puede efectuar.

trabajadores fuera de ella si se pudiera beneficiar del incremento de productividad resultante", Becker, G. S., p. 55.

15 Pierbattisti elabora un análisis iluminador sobre el valor de empleabilidad en oposición a la idea de "empleo de por vida". v. Pierbattisti, D. "Mecanismos disciplinarios, dispositivos de poder y neoliberalismo: formas de intervención sobre la fuerza de trabajo", en Herramienta. Revista de debate y crítica marxista. Buenos Aires, n. ${ }^{\circ} 34$, marzo, 2007. En este esquema encaja la nueva caracterización del desempleado como "buscador de empleo" que continúa trabajando en el incremento de su empleabilidad.

16 "Una inversión implica que se comprometen recursos para la adquisición de ingresos y satisfacciones futuras" Schultz, T. W. Invirtiendo en la gente, op. cit., p. 133. La dinámica de la inversión en sí misma posee un ritmo propio. En esta medida, las oportunidades de inversión y el nivel de rendimiento esperado van a estar en estrecha relación con el estado del capital que es el sujeto mismo. El sujeto como máquina que produce flujo de ingresos "se desvaloriza con el tiempo y siguiendo una tasa creciente a medida que avanza la vida" Schultz, T. W., p. 21. 
El caso de la educación y la formación universitaria constituye uno de los focos de Becker y Schultz por incidir en los elementos adquiridos que configuran e incrementan de forma voluntaria un determinado capital humano. Este permite sacar a relucir lo que ellos consideran un desfase en el planteamiento económico clásico: el consumo en una economía de la competencia consiste en una "actividad de empresa" que posibilita al sujeto producir algo. La mutación de la teoría del consumo ha abierto un nuevo campo de análisis en torno al comportamiento de los sujetos como pequeñas empresas. Inspirado en esta idea, Becker dedica toda una obra a la lógica que guía el comportamiento de los hogares, concluyendo que los bienes adquiridos en el mercado pasan a integrarse en la actividad productora de la familia como empresa (Becker, 1987, pp. 22-23). En este sentido Schultz afirma que “[...] es un grave error tratar todos los desembolsos educacionales como consumo corriente" (Becker, 1987, p. 101). La escolarización no puede igualarse a una actividad de consumo que satisface y beneficia al inversor en el lapso de tiempo que dura la actividad; ha de comprenderse como la inversión en un proyecto a largo plazo. Esto quiere decir que, considerada a corto plazo, la inversión en educación es vista como el desembolso de una cantidad de capital (tanto físico como intangible) que cae al vacío. Por el contrario, señalan Becker y Schultz, a propósito de la educación universitaria, que cuando el rendimiento es evaluado con referencia a las ganancias salariales absolutas (y no la diferencia relativa) se visibiliza la potencialidad que conlleva esta forma de inversión, ahora comprendida en términos de "adiciones al stock"17 (Schultz, 1985, p. 48).

Se delinea el trazo que une la inversión en educación, la productividad económica y, por último, el incremento del bienestar general. En la sociedad de los competentes, por lo tanto, “[...] los estudiantes asignan tanto su tiempo como los servicios educacionales que compran, en respuesta a los cambios en las ganancias esperadas [...]" (Schultz, 1985 , p. 33 $)^{18}$. Los incentivos para invertir son susceptibles de un análisis racional, donde la ganancia económica y psicológica que vaya a recibir el inversor constituyen dos de los criterios esenciales. Así se completa el círculo de la neoliberalización; la autorresponsabilidad como cifra de este ethos empresarial cierra el nudo.

17 "Los costes incluyen el valor asignado al tiempo y al esfuerzo de los aprendices, la "enseñanza" que otros proporcionan y el equipo y los materiales que se utilizan. [...] Estas partidas son costes, porque de no haberse utilizado para incrementar la producción futura podrían haber sido utilizadas para obtener producto en el presente" Becker, G. S. El capital humano, op. cit., p. 29. 


\section{Producción inmaterial y capital intelectual}

La consideración de la cuestión fundamental de la libertad en su análisis condujo a Foucault a revisar lo que hasta el momento había entendido por disciplina. El autor de Poitiers ya se percató de la necesidad de nuevas formas de control, vigilancia y gestión de este nuevo capital que se caracteriza por su inmaterialidad. El objetivo, al igual que lo era en el caso de la sociedad disciplinaria, sigue siendo el de la maximización del beneficio económico y de los niveles de productividad. En el contexto del New Deal y el keynesianismo las exigencias quedaban resumidas en contar, asistir y controlar ante el problema fundamental de la época: la insubordinación del trabajador. A este respecto resulta ilustrativa la exposición de Coriat $(2001)^{19}$ :

El cronómetro ha entrado en el taller: indudablemente está en marcha la mayor revolución de la historia humana. [...] Con esto también se inicia una secuencia económica enteramente nueva, un modo y un régimen nuevos de acumulación del capital: surge la producción en masa.

Finalmente, con la producción en masa y como condición de esta, se inicia una nueva secuencia en las modalidades y las prácticas estatales de regulación y control social (pp. 2-5).

El Estado correlativo a estas exigencias del nuevo modelo de producción queda definido como operador general de la reproducción del trabajo asalariado. Coriat considera que en este momento se produjo el primer cruce entre imperativos económicos e imperativos políticos, contacto que se materializa en la aparición de ciertos dispositivos materiales y legislativos donde el Estado, -mitad "providencia", mitad policía-, se sitúa a medio camino entre capital y sociedad civil. En efecto, el nuevo modelo de producción en masa requería una nueva organización y naturaleza de las práctica estatales de regulación y control social (Coriat, 2001, pp. 5-6).

El paso de una disciplina de los cuerpos a una regularización y normalización del "alma" del trabajador puede ser identificada a propósito de los efectos que la introducción de nuevas máquinas de trabajo tuvo sobre el trabajador. La aparición del

19 Coriat específica, y esto es importante, que cuando dice "taller" está refiriéndose a cualquier espacio en el que se inserte la actividad de trabajo. 
fair play (salario justo), que en sus inicios tenía por objetivo apartar al obrero de los sindicatos y así evitar las consecuentes distorsiones en la política laboral, se transfigura en el "instrumento de reproducción del trabajador"20 (Coriat, 2001, p. 53). En efecto, "la máquina no solo posee la virtud "económica" de hacer el trabajo más productivo, sino que sobre todo [...] puede ser instrumento de "regularización" y sometimiento de los trabajadores" (Coriat, 2001, p. 17). Un ejemplo de los mecanismos regularizadores puestos en marcha con la detección del peso que para el mercado tiene la actividad del trabajador es el denominado five dollars day. Este mecanismo "instaura, mediante el control del gasto del salario, una influencia en las condiciones de existencia de la población obrera" (Coriat, 2001, p. 27). Esto implica la agregación de nuevos requisitos que ha de cumplir el trabajador, desde la "limpieza" hasta la exigencia de "una moral intachable". Los requisitos en el acceso al puesto de trabajo dependerán a su vez de las exigencias que en cierto periodo imponga la naturaleza del capital. Actualmente lo que en el lenguaje empresarial se denomina como "prospección y proceso de selección" tiene por función la definición y clasificación del capital humano que requiere un determinado proyecto. En esta fase valorativa no entran en consideración de un modo exclusivo aspectos correspondientes a ciertas habilidades y saberes técnicos que el futuro empleado vaya a aplicar en el puesto de trabajo, sino aspectos que conciernen a la propia subjetividad del "buscador de empleo". Cuando la empresa deja de ser meramente el lugar de trabajo, y la vida diaria ingresa en el mercado adoptando la racionalidad económica, se produce una entera remodelación de la existencia misma del sujeto.

Esta mutación del capital tiene un precedente en la crisis del taylorismo y el agotamiento de la organización científica del trabajo fordiana. El acelerado ritmo de desarrollo del capitalismo instó a los intelectuales de la época a buscar "nuevas "palancas" susceptibles de incrementar la productividad e intensidad del trabajo" (Coriat, 2001, p. 154). La consideración del trabajo no ya como fuerza de trabajo sino como capital (humano) e idoneidad se encuadra en la progresiva modernización de la producción en el capitalismo. Lo "invisible" del trabajo es llamado a comparecer en la actividad económica, por cuanto posee efectos directos en el logro de los fines de productividad (Coriat, 1993, p. 179). Cualquier avance que se considere estrictamente tecnológico conlleva un segundo aspecto igualmente esencial: una transfiguración en el uso y las

20 En este sentido, "el efecto del incremento de los salarios sobre la productividad depende del uso a que se dediquen estos. [...] Las empresas pueden influir sobre el gasto induciendo a sus empleados" a que consuman adecuadamente ciertos bienes. Becker, G. S. El capital humano, op. cit., p. 57. 
modalidades de consumo productivo del trabajo vivo. En el umbral de la metamorfosis del trabajo en una economía de la competencia, se saca a relucir el carácter limitado de las innovaciones propiamente tecnológicas, al mismo tiempo que cobra fuerza la hipótesis de que "la competitividad calidad se funda centralmente en la calificación del trabajo, la inversión organizacional y la valorización sistemática de los recursos humanos" (Coriat, 1993, p. 224).

Es conveniente aportar un breve apunte sobre la inversión organizacional que hemos mencionado. Teniendo en cuenta que "[...] las sociedades disciplinarias son nuestro pasado inmediato, lo que estamos dejando de ser" (Deleuze, 1996, p. 278), el rasgo flexible y funcional de los nuevos puestos de trabajo corresponde al camino iniciado en la dirección de la eliminación de la rígida línea que separaba al obrero del patrono, y esto porque "[...] el trabajo libre es más productivo que el servil [...]" (Mises, 1995, p. 39). Esto no significa, sin embargo, que los mecanismos de control pasen a ser menos densos; ahora es el individuo el que ejerce sobre sí mismo un autocontrol omnipresente, resultando de ello que "la "autonomía" se convierte en instrumento de autodisciplina” (Coriat, 2001, p. 177). La mutación epistemológica del capital y del trabajo se ha refinado progresivamente conforme a los desarrollos en el nivel de la organización de la producción. Con el abandono del dispositivo fordiano se abre paso a una nueva economía del tiempo y del control. Frente al control individualizado de cada trabajador, las técnicas de vigilancia sufren una modificación: la supervisión del trabajo y de los empleados se lleva a cabo en una forma mucho más sutil, económica y eficaz. El control del tiempo global del trabajo en grupo hace ingresar al empleado en una red de relaciones con el resto de compañeros de trabajo, codificadas desde ese momento como una relación entre clientes internos a la empresa (Coriat, 2001, pp. 176-177). La garantía de un ejercicio responsable de esta libertad que se otorga al empleado es lo que el departamento de RR.HH de una empresa trata de articular: un modelo de gestión humanística. Por humanismo entienden la consideración del empleado en tanto que persona; esto es, como agente económico activo cuya conducta, susceptible de análisis económico, ha de ser encauzada por la senda de la eficiencia y la productividad proporcionando un entorno preciso para su estímulo (Pascual, 2013, p. 141). La presencia "metaestable" de la empresa y de los procesos de formación culmina en el establecimiento de una suerte de continuidad que borra finalmente la delimitación entre los diversos ámbitos de la vida del individuo (Deleuze, 1996, p. 280). Es así como en las sociedades de control se constituye una modulación constante del individuo, a la que Deleuze se referirá como "moldeado 
autodeformante" que, a su vez, cambia constantemente en correspondencia con los movimientos propios de la empresa:

La fábrica hacía de los individuos un cuerpo, con la doble ventaja de que, de este modo, el patrono podía vigilar cada uno de los elementos que formaban la masa y los sindicatos podían movilizar a toda una masa de resistentes. La empresa, en cambio, instituye entre los individuos una rivalidad interminable a modo de sana competición, como una motivación excelente que contrapone unos individuos a otros y atraviesa a cada uno de ellos, dividiéndole interiormente (Deleuze, 1996, p. 280).

No ha de extrañar la aparición en esta área de expresiones como "gestión y rentabilidad de la felicidad", "gestión del talento", incluso "gestión del alma" de las empresas, siendo las personas, en efecto, la sustancia de esta ${ }^{21}$.

El capital, en la necesidad de ajustarse a la dinámica del mercado, sufrió una segunda mutación. A rasgos generales, el capital en el que actualmente reside la "riqueza de una nación" es el capital intelectual. Lo intangible se torna, finalmente, en el elemento determinante de la realidad en su continua materialización: la información y el conocimiento tienen vida económica (Stewart, 1998, p. 64). Por capital intelectual se comprende un "material intelectual -conocimientos, información, propiedad intelectual, experiencia- que se puede aprovechar para crear riqueza” (Stewart, 1998, p. 10). En esta medida, la conducta maximizadora con la que los economistas definían al sujeto, se aplica aquí con la misma fuerza. En efecto, comienzan a proliferar una serie de discursos (en el ámbito económico) que intentan establecer las condiciones que determinen la caracterización y clasificación de los conocimientos importantes, rentables (Stewart, 1998, p. 38).

Empresa intelectual y trabajo intelectual constituyen el topos del mundo laboral actual. En este contexto, la inversión en educación se revela como factor fundamental en la

21 Nos sorprende especialmente la expresión "gestión del alma". Esta aparecía ya en los albores del liberalismo como eje vertebrador de los cambios económicos y sociales. La interioridad del sujeto es de nuevo arrastrada al exterior y puesta bajo custodia en el mercado laboral; así lo quiere el discurso empresarial. Los incentivos para invertir en capital humano van a tener este aspecto muy presente: "la mejora de la salud fisica y psíquica es un tipo de inversión en capital humano [...] la estabilidad emocional se considera cada vez más como un determinante fundamental de las retribuciones [...]". Becker, G. S. El capital humano, op. cit., p. 54. 
creación de valor y riqueza. Esto implica que el valor del conocimiento va a estar en estrecha relación con su utilidad, siendo así que el efecto en las retribuciones futuras pasa a constituir el valor de medida:

\begin{abstract}
El espíritu empresarial también es esencial en la investigación, que es siempre una empresa arriesgada, que impone la necesidad de organizar y distribuir recursos escasos. La esencia misma de la investigación consiste en una aventura dinámica hacia lo desconocido o solo a medias conocido. [...] Alguien debe decidir cómo se han de distribuir, dado el estado actual del conocimiento, los limitados recursos de que se dispone (Schultz, 1985, p. 18. Cursiva propia).
\end{abstract}

El valor del capital intelectual que el sujeto "lleva consigo entre oreja y oreja" queda cifrado en el carácter innovador y creador. Este, imbricado con el ethos empresarial que el empleado ha integrado en la configuración de su propia subjetividad, es el verdadero factor del desarrollo del mercado. Como afirma Stewart, "de nada sirve tener un tipo muy sabio sentado a solas en una habitación” (Stewart, 1998, p. 127).

A partir de lo dicho puede comprenderse la función social que juegan las escuelas y las universidades en nuestro presente. Las universidades, entendidas como "empresas multiproducto" especializadas en la producción de formación, ofertan "un conjunto de cualificaciones" que han de considerarse valiosas en el mercado (Becker, 1983, p. 214). Esto permite sostener a los economistas que: “[...] no siempre es necesario hacer una distinción tajante entre escuelas y empresas: en algunos casos se puede considerar a las escuelas como un tipo especial de empresa, y a los estudiantes como un tipo especial de aprendiz" (Becker, 1983, p. 51). Las mutaciones epistemológicas a las que nos hemos referido han derivado finalmente en la integración del pensamiento en el mercado. Competencia, aprendizaje y pensamiento se tornan indiscernibles en esta dinámica a la que el sujeto empresario de sí debe ajustarse. Lo más importante a nuestro juicio consiste en el progresivo (y sutil) proceso de sometimiento en el que el sujeto se ha visto arrastrado. El carácter innovador y creativo del sujeto, supuestamente autónomo tal y como los neoliberales gustan llamar, queda subsumido en el esquema de principios y fines. Esta cultura empresarial que define a todos y cada uno de los sujetos puede resumirse en los siguientes consejos y advertencias:

No le ofrecemos estabilidad, pero en este trabajo exigente usted puede adquirir nuevas destrezas que aumentan su valor en el mercado. Actúe como si fuera un cuentapropista, un empleado de Yo Mismo S. A. Todos los trabajos ofrecidos 
son temporarios. Usted es el único responsable de manejar su propia carrera (Stewart, 1998, p. 290).

Cuando Foucault implementa sus análisis genealógicos con las nuevas regulaciones biopolíticas, creemos ver, no abandona los mecanismos disciplinarios como instrumento en el desciframiento del diagrama de poder. Los mecanismos de control y los procesos de subjetivación, haciendo de las mentes una materia dócil, han logrado responder a las exigencias fijadas por los movimientos del mercado, cumpliendo el único imperativo incondicional sobre el que se articula nuestro tiempo: incrementar e implementar el capital humano que es el propio sujeto con el fin de que la economía de mercado pueda seguir su propio camino.

\section{Referencias}

Becker, G. S. (1983). El capital humano. Madrid: Alianza.

Becker, G. S. (1987). Tratado sobre la familia. Madrid: Alianza.

Coriat, B. (1993). El taller y el robot. Ensayos sobre el fordismo y la producción en masa en la era de la electrónica. Madrid: Siglo XXI.

Coriat, B. (2001). El taller y el cronómetro. Ensayo sobre el taylorismo, el fordismo y la producción en masa. Madrid: Siglo XXI.

Deleuze, G. (1996). Post-scriptum sobre las sociedades de control. En Pardo, J. L. (Trad.). Conversaciones 1972-1990. Valencia: Pre-Textos.

Febrero, R., y Schwartz, P. (Eds.). (2001). La visión personal de Becker. El discurso del Nobel: el modo económico de analizar el comportamiento. La esencia de Becker. Barcelona: Ariel.

Foucault, M. (2007). Nacimiento de la biopolítica. Buenos Aires: FCE.

Hayek, F. A. (1976). Law, legislation and liberty, vol. II. Chicago: The University of Chicago Press. 
Hayek, F. A. (1981). Los fundamentos éticos de una sociedad libre. Ciclo de conferencias sobre Fundamentos de un Sistema Social Libre. Santiago de Chile: Centro de Estudios Públicos.

Huerta, J. (1997). La escuela austríaca: mercado y creatividad empresaria. Madrid: Instituto de Análisis Industrial y Financiero: UCM.

Matsusaka, J. G. (1995). The economic approach to democracy. En Tommasi, M., y Ierulli, K. (Eds.). The new economics of human behavior. Cambridge: Cambridge University Press.

Mises, L. (1995). Sobre liberalismo y capitalismo. Reig, J. (Trad.). Madrid: Unión Editorial.

Montag, W. (2006). El peligroso derecho a la existencia: la necroeconomía de Von Mises y Hayek. En Youkali. Revista crítica de las artes y el pensamiento, (2).

Morales-Arce, R. (2005). En torno al capital humano: aspectos básicos en su consideración empresarial. Madrid: Centro de Estudios Ramón Areces.

Pascual, J. L. (2013). Gestión del capital humano en las organizaciones: entre nichos y bichos, ¿valor estructural o valor emocional? Tarragona: Altaria.

Pierbattisti, D. (2007, marzo). Mecanismos disciplinarios, dispositivos de poder y neoliberalismo: formas de intervención sobre la fuerza de trabajo. en Herramienta. Revista de debate y crítica marxista, (34).

Röpke, W. (1971). Estado benefactor e inflación crónica (trad. al español). A Humane Economy: The social framework of the free market. Chicago: Henry Regnery Company, A Gateway Edition.

Rose, N. (1996). Governing "advanced" liberal democracies. En Barry, O. R. Foucault and political reason. Liberalism, neoliberalism and rationalities of government. London: UCL Press.

Schultz, T. W. (1985). Invirtiendo en la gente: la cualificación personal como motor económico. Barcelona: Ariel, S. A.

Stewart, T. A. (1998). La nueva riqueza de las organizaciones: el capital intelectual. Buenos Aires: Granica. 
Ugarte, J. (2006). Biopolítica. Un análisis de la cuestión. En Claves de razón práctica, (166). Madrid.

Vázquez, F. (2005). Empresarios de nosotros mismos. Biopolítica, mercado y soberanía en la gubernamentalidad neolibera. En Ugarte, J. (De.). La administración de la vida. Estudios biopolíticos. Barcelona: Anthropos. 